\title{
Cuidados fornecidos por familiares relacionados à convivência com o idoso
}

\author{
Caring services provided by relatives related to \\ elderly interaction \\ Cuidados suministrados por familiares relacionados \\ a la convivencia con el anciano
}

Luisa Kecyane Batista Cardoso

Talita Santos Oliveira Sampaio

Alba Benemérita Alves Vilela

RESUMO: Este estudo teve como objetivo identificar os cuidados fornecidos pelos familiares relacionados à convivência com o idoso, cadastrados em uma USF da cidade de Jequié, BA. Encontrou-se que os cuidados são relacionados principalmente à alimentação e às medicações, preocupando-se pouco com os hábitos de vida. Concluiuse que existe uma deficiência no conhecimento dos cuidadores/familiares acerca de cuidados necessários voltados à convivência com o idoso no contexto familiar.

Palavras-chave: Idoso; Relações Familiares; Saúde Pública.

ABSTRACT: This study aimed to identify the caring services provided by family members related to interaction with the elderly, registered in a USF in the city of Jequié, BA. It was found that caring services is mainly related to food and medications, worrying less about the life habits. It was concluded that there is a lack of knowledge of caregivers / relatives about necessary care focused on living with elderly in the family context.

Keywords: Aged; Family Relations; Public Health. 
RESUMEN: Este estudio tuvo cómo objetivo identificar los cuidados suministrados por los familiares relacionados a la convivencia con el anciano, dados de alta en una USF de la ciudad de Jequié, BA Se encontró que los cuidados son relacionados principalmente la alimentación y las medicações, preocupándose poco con los hábitos de vida. Se concluyó que existe una deficiencia en el conocimiento de los cuidadores/familiares acerca cuidados necesarios vueltos a la convivencia con anciano en el contexto familiar

Palabras clave: Anciano; Relaciones Familiares; Salud Pública.

\section{Introdução}

O desenvolvimento tecnológico e terapêutico, as quedas na taxa de fecundidade e mortalidade, e as melhorias nas condições de saúde, são fatores que influenciaram no crescente aumento da população idosa mundial. Primeiramente esse fenômeno ocorreu em países desenvolvidos; no entanto, atualmente os países em desenvolvimento apresentam de forma mais intensa e acelerada o envelhecimento populacional. No Brasil, o número de pessoas com 60 anos ou mais representam 10,8\% da população. As estatísticas sugerem que, em 2025, o Brasil deverá ter 15\% de sua população constituída por idosos, tornando-se assim o país em sexto lugar no ranking mundial (Brasil, 2010; Smith, et al., 2011).

O efeito do envelhecimento da população provoca mudanças significativas no processo de transição epidemiológica, e também nos padrões de morbimortalidade. Observa-se uma prevalência de doenças crônico-degenerativas, que promovem um aumento da fragilidade e incapacidades físicas e mentais no idoso (Santos, \& Tavares, 2012; Gratão, et al., 2013). Somado à presença dessas doenças, há ainda um declínio funcional próprio do processo de envelhecimento que altera a qualidade de vida, dificultando a realização de tarefas que antes eram feitas com facilidade, fazendo com que o idoso apresente dependência funcional para realização de suas atividades básicas da vida diária (Oliveira, \& Tavares, 2014).

Pesquisas mostram que cerca de $40 \%$ da população idosa necessita de algum tipo de auxílio para realizar pelo menos uma Atividade Instrumental da Vida Diária (AIVD), como por exemplo, cuidar das finanças, utilizar transportes, cozinhar, entre outras. 
Além disso, existem pessoas idosas que também necessitam de ajuda para realizar Atividades Básicas de Vida Diária (AVD), sendo relacionadas ao autocuidado, alimentação, higiene pessoal, mobilidade e vestir-se (Gratão, et al., 2013).

Em decorrência desse cenário, observa-se a necessidade cada vez maior pelos idosos de suporte familiar. A família é vista como a base para garantir o sustento, desenvolvimento e proteção de seus membros, independentemente da forma como ela está estruturada (Monteiro, \& Sena, 2012). Segundo o Estatuto do Idoso, criado em 2003, é obrigação da família, da comunidade, da sociedade e do Poder Público assegurar ao idoso, com absoluta prioridade, a efetivação do direito à vida, à saúde, à alimentação, à educação, à cultura, ao esporte, ao lazer, ao trabalho, à cidadania, à liberdade, à dignidade, ao respeito e a convivência familiar e comunitária (Brasil, 2003).

As famílias têm passado por algumas transformações na sua estrutura, tornandose nucleares, com rearranjos visando ao atendimento das necessidades dos membros mais velhos e dependentes, neste novo contexto, com destaque para a família intergeracional. Neste tipo de arranjo, o idoso convive com múltiplas gerações no mesmo espaço domiciliar, caracterizando, dessa forma, a corresidência (Santos, \& Pavarini, 2012; Camarano, Kanso, Mello, \& Pasinato, 2004).

O contexto familiar também é considerado um sistema de saúde para os idosos, uma vez que seus membros apresentam conhecimentos e práticas voltados para a prevenção e o tratamento de doenças. Esse suporte familiar de cuidados favorece a saúde física e psicológica; entretanto, seu efeito é tido como benéfico, se executado pelos membros da família de forma satisfatória e com responsabilidade. Sendo assim, seria necessário indagar, segundo Costa e Lodovici (2016), os modos de operação (modus operandi) das práticas cotidianas de um cuidador familiar principal. ${ }^{1}$ Há também a necessidade de uma assistência adequada ao próprio idoso, assim como a seus familiares, pelos profissionais de saúde, numa abordagem que insira a família no planejamento das ações de cuidado, buscando-se compreender como esses familiares lidam com o envelhecimento de seus entes queridos (Oliveira, Boaretto, Vieira, \& Tavares, 2014).

\footnotetext{
1 “Cuidador familiar principal: aquele que, em uma família, assume deliberadamente, ou por designação dos demais membros, ou por sua própria delegação ou omissão, o cuidado com uma pessoa doente, em casa." (Costa, \& Lodovici, 2016, p. 33). 
Destarte que existem cuidados sistematizados e necessários que precisam ser de conhecimento do cuidador/familiar que reside com o idoso, buscando-se a qualidade de vida e uma convivência harmoniosa entre idosos e familiares. Entretanto, sabe-se que ainda existe certa escassez de conhecimentos dos familiares sobre os cuidados com a pessoa idosa em corresidência.

Diante dessa realidade na qual estamos inseridos, este estudo tem por objetivo conhecer a percepção dos familiares acerca dos cuidados necessários voltados à convivência com o idoso. Os resultados obtidos no presente trabalho serão disseminados no meio científico, com intuito de contribuir para a promoção de planejamento e ações capazes de assistir as demandas de idosos e seus familiares.

\section{Método}

Trata-se de um estudo de característica exploratória e descritiva, com delineamento transversal, e abordagem quantitativa. $\mathrm{O}$ estudo foi realizado em uma Unidade de Saúde da Família (USF) do município de Jequié, BA, sendo escolhida por meio de um sorteio simples.

A população do estudo foi constituída por familiares, que corresidem com os idosos cadastrados na área de abrangência da referida USF, residentes na zona urbana, totalizando 133 idosos. Após o contato com os Agentes Comunitários de Saúde (ACS) que fazem parte dessa USF e, levando-se em consideração os critérios de inclusão e exclusão, participaram do estudo familiares de 40 idosos. Esses idosos encontravam-se em estado de corresidência com seus familiares, durante o período de março a junho de 2014.

A coleta de dados ocorreu por meio de visitas domiciliares com auxílio dos ACS das microáreas adstritas a USF em estudo, para melhor proporcionar o contato com o ambiente familiar no qual o idoso está inserido. Aplicou-se um questionário semiestruturado aos familiares cuidadores, contendo 26 perguntas relativas às condições sociodemográficas como idade, sexo, grau de parentesco com o idoso, renda familiar, bem como perguntas relacionadas aos cuidados prestados pelos familiares aos idosos. Para os idosos, foram feitas perguntas relativas à idade, sexo e necessidades de auxílios. 
Os dados obtidos foram armazenados em um banco de dados através do programa Microsoft Office Excel 2010 e, posteriormente, realizada a análise descritiva com base na distribuição de frequência simples e percentual.

Esta pesquisa obedeceu às normas éticas exigidas pela Resolução n. ${ }^{\circ}$ 466/2012 (Conselho Nacional de Saúde), que incluem a aplicação do Termo de Consentimento Livre e Esclarecido, TCLE assinado por cada participante, estando vinculada ao parecer n. 511.487 aprovada pelo Comitê de Ética e Pesquisa da Universidade Estadual do Sudoeste da Bahia.

\section{Resultados}

As características qualitativas da população estudada referentes às frequências entre os gêneros e idades dos idosos e dos familiares que se declararam cuidadores, são mostradas na Tabela 01. A maioria dos idosos apresenta idade entre 65 a 70 anos $(77,5 \%)$ e são do sexo masculino $(52,5 \%)$. Em relação aos familiares cuidadores, a maioria se encontra na faixa etária de 25 a 35 anos e são do sexo masculino (85,0\%).

Tabela 01. Análise descritiva das variáveis qualitativas referente às frequências de gêneros e idades dos idosos e dos familiares do estudo. Jequié, BA, Brasil, 2014

\begin{tabular}{l|l|l|l|l|l}
\hline \multicolumn{5}{l}{ IDOSO } & \multicolumn{1}{l}{ CUIDADOR } \\
\hline IDADE & Frequência & $\%$ & IDADE & Frequência & $\%$ \\
$=65$ anos & 04 & 10 & $>18-24$ & 06 & 15 \\
$65-70$ anos & 31 & 77.5 & $25-35$ & 24 & 60 \\
$>70$ anos & 05 & 12.5 & $>35$ & 10 & 25 \\
Total & 40 & 100 & Total & 40 & 100 \\
GÊNERO & 19 & 47,5 & GÊNERO & & \\
Masculino & 19 & 52,5 & Masculino & 06 & 15 \\
Feminino & 21 & 100 & Teminino & 34 & 85 \\
Total & 40 & Total & 40 & 100 \\
\hline
\end{tabular}

Fonte: Dados da pesquisa

A tabela 02 apresenta o grau de parentesco dos familiares que se declararam cuidadores com relação ao idoso em corresidência. Observou-se que a maior parcela dos familiares possui o grau de parentesco de filhos (55,0\%), seguido de esposas(os) $(40,0 \%)$. 
Tabela 02: Grau de parentesco dos cuidadores/familiares dos idosos. Jequié, BA, Brasil, 2014

\begin{tabular}{l|l|l}
\hline Grau de parentesco & Frequência & \% \\
\hline Filhos & 22 & 55 \\
Esposa (os) & 16 & 40 \\
Nora & 01 & 2,5 \\
Netos & 01 & 2,5 \\
Total & 40 & 100 \\
\hline
\end{tabular}

Fonte: Dados da pesquisa

Na tabela 03 , verifica-se a distribuição da renda familiar, onde $(55,0 \%)$ possuem uma renda mensal de 1 a 3 salários mínimos, (40,0\%) responderam que a renda da família se refere a 4 a 6 salários mínimos e acima de sete salários, apenas $(6,25 \%)$.

Tabela 03: Concentração da renda familiar. Jequié, BA, Brasil, 2014

\begin{tabular}{l|l|l}
\hline & Frequência & $\%$ \\
\hline 1 a 3 salários mínimos & 22 & 55 \\
4 a 6 salários mínimos & 16 & 40 \\
Acima de 07 salários & 02 & 6,25 \\
Total & 40 & 100 \\
\hline
\end{tabular}

Fonte: Dados da pesquisa

Os dados da tabela 04 apresentam o principal responsável pela renda familiar. Dentre os resultados, encontrou-se que (32,5\%) dos idosos são os principais responsáveis pela renda da família, e em $(25,0 \%)$ o principal responsável é o idoso juntamente com o cônjuge.

Tabela 04: Principal responsável pela renda familiar. Jequié, BA, Brasil, 2014

\begin{tabular}{l|l|l}
\hline Principal responsável & Frequência & $\%$ \\
\hline Idoso & 13 & 32,5 \\
Idoso e cônjuge & 10 & 25 \\
Família & 04 & 10 \\
Idoso e fillhos & 07 & 17,5 \\
Outros & 06 & 15 \\
Total & 40 & 100 \\
\hline
\end{tabular}

Fonte: Dados da pesquisa 
A tabela 05 apresenta dados sobre a necessidade do idoso de possuir um cuidador para ajudar nas suas atividades do cotidiano. Pode-se observar que $(70,0 \%)$ dos idosos relataram apresentar necessidade de ajuda no cotidiano e $30 \%$ afirmaram que são responsáveis pela sua própria saúde e bem-estar, sendo que, em alguns momentos necessitam de ajuda para realização de suas atividades básicas da vida diária.

Tabela 05: Necessidade do idoso de possuir um cuidador para ajudar nas atividades do cotidiano. Jequié, BA, Brasil, 2014

\begin{tabular}{l|l|l}
\hline Necessitam de ajuda no cotidiano & Frequência & \% \\
\hline Sim & 28 & 70 \\
Não & 12 & 30 \\
Total & 40 & 100 \\
\hline
\end{tabular}

Fonte: Dados da pesquisa

Na tabela 06, observa-se a distribuição da relação dos cuidados prestados pelos familiares aos idosos. Percebe-se que o cuidado com o preparo das refeições foi o mais citado (72,5\%), seguido de cuidados relacionados com as medicações $(22 \%)$.

Tabela 06: Relação dos cuidados prestados pelos familiares. Jequié, BA, Brasil, 2014

\begin{tabular}{c|l|l}
\hline Categorias & Frequência & \% \\
\hline 1- $\begin{array}{l}\text { Cuidados com as } \\
\text { medicações }\end{array}$ & 09 & 22 \\
2- $\begin{array}{l}\text { Cuidados com o preparo } \\
\text { das refeições }\end{array}$ & 29 & 72,5 \\
3- Cuidados relacionados ao & 08 & \\
lar & & 20 \\
4- Servir de companhia & 09 & 22,5 \\
5- Acompanhar nas consultas & 03 & 7,5 \\
6- Lazer & 07 & 17,5 \\
7- Nenhum & 01 & 2,5 \\
\hline
\end{tabular}

Fonte: Dados da pesquisa

A tabela 07 apresenta os dados referentes à responsabilidade no preparo das refeições. Observou-se que, na dinâmica familiar, o idoso é o principal responsável pelo preparo das refeições $(40,0 \%)$, seguido pelos cônjuges $(35,0 \%)$ e $(25,0 \%)$ dos familiares ou o cuidador/familiar. 
Tabela 07: Responsabilidade no preparo das refeições. Jequié, BA, Brasil, 2014

\begin{tabular}{l|l|l}
\hline $\begin{array}{l}\text { Responsável em preparar a } \\
\text { refeição }\end{array}$ & Frequência & \% \\
\hline Idoso & 16 & 40 \\
Cônjuge & 14 & 35 \\
Familiares ou & & \\
Cuidador/familiar & 10 & 25 \\
Total & 40 & 100 \\
\hline
\end{tabular}

Fonte: Dados da pesquisa

A tabela 08 apresenta ainda informações no que diz respeito aos cuidados com a alimentação dos idosos. Verifica-se que $(85,05 \%)$ dos entrevistados relataram cuidados relativos à diminuição do sal e $(65,0 \%)$ referiram cuidados em relação à diminuição da gordura.

Tabela 08: Principais cuidados com as refeições. Jequié, BA, Brasil, 2014

\begin{tabular}{l|l|l}
\hline $\begin{array}{l}\text { Cuidados relacionados com } \\
\text { o preparo das refeições }\end{array}$ & Frequência & $\%$ \\
\hline $\begin{array}{l}\text { Diminuir o consumo do sal } \\
\text { de cozinha }\end{array}$ & 34 & 65 \\
$\begin{array}{l}\text { Diminuir o consumo de } \\
\text { gordura }\end{array}$ & 26 & 57,5 \\
$\begin{array}{l}\text { Aumentar o consumo de } \\
\text { verduras }\end{array}$ & 23 & 40 \\
$\begin{array}{l}\text { Incentivar o idoso a consumir } \\
\text { mais frutas }\end{array}$ & 16 & 12,5 \\
Consumo de azeite & 05 & 22,5 \\
Diminuição do consumo de & 09 & \\
açúcar &
\end{tabular}

Fonte: Dados da pesquisa 
$\mathrm{Na}$ tabela 09, encontra-se a informação sobre a realização da prática de exercícios físicos pelos idosos em corresidência. Observa-se que $(65,0 \%)$ dos idosos não exercem nenhum tipo de atividade física e apenas $(35,0 \%)$ referiram praticar exercícios físicos.

Tabela 09: Prática de exercícios físicos entre os idosos. Jequié, BA, Brasil, 2014

\begin{tabular}{l|l|l}
\hline $\begin{array}{l}\text { Exercem algum tipo de } \\
\text { atividade física }\end{array}$ & Frequência & $\%$ \\
\hline Sim & 14 & 35 \\
Não & 26 & 65 \\
Total & 40 & 100 \\
\hline
\end{tabular}

Fonte: Dados da pesquisa

A tabela 10 retrata a relação dos indivíduos que mais estimulam os idosos a praticarem algum tipo de exercício físico diário, segundo os familiares. 25,0\% relataram que apenas o enfermeiro da Unidade de Saúde da Família realiza esse incentivo e 17,5\% afirmaram ser a própria família, os principais incentivadores da prática de atividades físicas.

Tabela 10: Relação dos indivíduos que mais estimulam os idosos a praticarem algum tipo de atividade física. Jequié, BA, Brasil, 2014

\begin{tabular}{l|l|l}
\hline Responsável & Frequência & \% \\
\hline Apenas a Família & 07 & 17,5 \\
Apenas o Médico & 05 & 12,5 \\
Família e médico & 06 & 15 \\
Apenas o Enfermeiro & 10 & 25 \\
Enfermeiro e médico & 05 & 12,5 \\
Apenas os amigos & 02 & 05 \\
Ninguém influencia & 05 & 12,5 \\
Total & 40 & 100 \\
\hline
\end{tabular}

Fonte: Dados da pesquisa

Na tabela 11, observam-se dados relativos à presença de orientações por parte de algum profissional de saúde sobre os cuidados relativos aos idosos, recebidos pelos familiares cuidadores. Observa-se que $(65,0 \%)$ dos familiares afirmaram receber orientação sobre os cuidados prestados aos idosos por algum profissional de saúde. 
Dentre estes, 50,0\% relataram o enfermeiro como o profissional de saúde que mais realiza essas orientações.

Tabela 11: Relação dos familiares que já receberam ou não orientações de algum profissional de saúde. Jequié, BA, Brasil, 2014

\begin{tabular}{llllll}
\hline Já recebeu & Frequência & $\%$ & De quem & Frequência & $\%$ \\
\hline Sim & 26 & 65 & -Médico & 09 & 34,61 \\
& & & -Enfermeiro & 13 & 50 \\
& & & - ACS & 03 & 11,53 \\
& & & - Outros & 01 & 3,84 \\
Não & 14 & 35 & - & - & - \\
Total & 40 & 100 & & 26 & 100 \\
\hline
\end{tabular}

Fonte: Dados da pesquisa

\section{Discussão}

No que se refere às características sociodemográficas dos idosos, observa-se que, dentre os idosos entrevistados, prevaleceu o gênero feminino (52,5\%). As mulheres apresentam uma melhor expectativa de vida em relação aos homens, devido a vários fatores como, por exemplo, embora declarem mais doenças que os homens, se cuidam, porém, mais. Também existe a questão dos anos adicionais que é maior nas mulheres do que nos homens (Camargos, Rodrigues, \& Machado, 2009).

Em relação à renda familiar, os resultados demonstram que a renda se concentra entre 01 a 03 salários mínimos, e as despesas da casa são compartilhadas entre homens e mulheres, ou o próprio idoso é o único responsável. A renda familiar tem função importante na corresidência, pois é ela que garante sustento para todos os componentes da família. Uma renda que é recebida pelo idoso poderá estimulá-lo a se tornar independente, mas, por outro lado, também poderá atrair familiares que desejam compartilhar desse benefício, aumentando a dependência, muitas vezes, da família em relação ao idoso (Edmonds, Mammen, \& Miller, 2005; Ghosh, 2007; Marteleto, 2007). 
No presente estudo, foi constatado que a maioria dos idosos necessita de algum tipo de ajuda. Esse auxílio é fornecido, na maioria das vezes, pelos familiares principalmente em corresidência, sendo os cuidadores do sexo feminino, os principais responsáveis pelo cuidado. Ainda nos dias atuais, a sociedade relaciona o papel do cuidar à mulher, devido a aspectos de ordem cultural (Vieira, et al., 2011).

Entende-se que o cuidador é a pessoa que oferece cuidados com o objetivo de suprir a incapacidade funcional, temporária, ou definitiva do idoso. $\mathrm{O}$ cuidado pode ser do tipo formal ou informal, sendo o cuidado formal fornecido pelo sistema de saúde, e o cuidado informal, incorporado pelos familiares, quando um deles assume a tarefa de ser o cuidador familiar. No Brasil, em relação aos idosos, prevalece o suporte prestado pelas famílias, principalmente porque há um número reduzido de instituições de longa permanência para idosos, e as que existem, muitas vezes, não possuem suporte adequado (Giacomin, Uchoa, \& Lima-Costa, 2005).

Observa-se que alguns dos idosos analisados sentem necessidade de um cuidador/familiar, devido à redução das funções cognitivas e físicas. $\mathrm{O}$ envelhecimento é um processo natural, quando ocorre a perda progressiva da funcionalidade dos indivíduos, que se somada à presença de doenças, estresse e outros motivos, podem ocasionar uma condição patológica que necessite de cuidador exclusivo (Brasil, 2006).

Os resultados do estudo mostram que a maioria dos familiares/cuidadores já receberam de algum profissional de saúde orientações e informações necessárias para melhorar a qualidade de vida do idoso em corresidência. Algumas atribuições dos profissionais de saúde envolvem oferecer atenção às necessidades de saúde da pessoa idosa, juntamente com os demais níveis de atenção, com vistas ao cuidado integral (Brasil, 2006).

Percebe-se que os cuidados mais comuns praticados estão relacionados ao preparo das refeições e controle do uso de medicações. Alguns estudos demonstram que ainda existem muitas dúvidas relacionadas aos cuidados com os idosos por parte desses familiares/cuidadores, como por exemplo, os cuidados com a casa para prevenção de quedas, questão que vem sendo pouco discutida (Freitas, Santos, Hammerschmidt, Silva, \& Pelze, 2011).

Outro problema encontrado no estudo refere-se ao elevado índice de idosos sedentários, e que, mesmo sendo estimulados à prática de exercícios, não há a efetivação dessa atividade. 
Ressalte-se que a prática de atividade física apresenta aspecto importante principalmente na prevenção de estados mórbidos como as doenças crônicodegenerativas, que estão relacionadas à presença de declínio funcional e cognitivo nos idosos (Madeira, et al., 2013; Mourão, Novais, Andreoni, \& Ramos, 2013).

Os principais novos desafios para o atual sistema de saúde no Brasil são representados pela prevenção das doenças crônico-degenerativas, uma melhor assistência à saúde dos idosos e ao suporte aos cuidadores familiares. Sendo assim, torna-se necessário o apoio aos familiares cuidadores para uma melhor utilização de habilidades e conhecimentos para diminuir ou até mesmo prevenir os efeitos secundários graves, físicos e/ou psicossocial que surgem em decorrência do envelhecimento (Santos, \& Pavarini, 2012). O cuidador/familiar tem papel fundamental no incentivo à prática de exercícios físicos, dieta balanceada e atividades de lazer, promovendo uma melhor qualidade de vida para o idoso (Santos, Kalsing, Ruiz, Roese, \& Gerhardt, 2013).

É preciso avaliar a funcionalidade familiar, pois não há certeza de que essa nova família esteja preparada para assumir a tarefa de cuidadora, mesmo sendo este cuidado realizado apenas no futuro. As famílias, muitas vezes, assumem os cuidados convivendo com dificuldades e sem orientação adequada para desenvolvê-los (Santos, \& Pavarini, 2012).

\section{Conclusão}

Considerando-se os resultados obtidos, foi possível observar que os idosos em corresidência recebem os cuidados mínimos para viver bem, em harmonia. Também se pode dizer que as orientações dadas pelos profissionais de saúde aos familiares não vêm sendo eficientes, dado que atentam somente para a alimentação e medicações, e não enfatizam os outros cuidados necessários. Também não se sabe se esses profissionais realizam visitas para avaliar se os cuidados com os idosos estão feitos de forma correta pelo cuidador/familiar.

Nota-se que nenhum idoso avaliado neste estudo dispõe de um cuidador que tenha passado por um curso específico para formação de cuidadores, inclusive o cuidador/familiar. Nenhum dos idosos pesquisados possuía cuidador contratado. 
Levando-se em consideração esses itens, vale salientar a necessidade de se estabelecerem políticas voltadas à capacitação desses familiares quanto aos cuidados e a convivência com o idoso.

Além disso, é necessário que a equipe multiprofissional da USF atue de forma integral a cuidadores e idosos, visando a melhorar a qualidade de vida de ambos.

A pesquisa conseguiu atender aos objetivos propostos, trazendo reflexões sobre como os cuidadores atuam para prevenir complicações à saúde do idoso, bem como eles se envolvem no processo de envelhecimento de um idoso de casa. Dessa forma, entende-se que seja necessário ampliar o conhecimento sobre as práticas voltadas à saúde do idoso, incluindo-se a promoção de saúde e a qualidade de vida.

\section{Referências}

Brasil. (2003). Ministério da Saúde. Estatuto do Idoso. Brasília, DF: Ministério da Saúde (70 p).

Brasil. (2006). Ministério da Saúde. Secretaria de Atenção à Saúde. Departamento de Atenção Básica. Envelhecimento e saúde da pessoa idosa. Brasília, DF (Cadernos de Atenção Básica, n. ${ }^{\circ}$ 19).

Brasil. (2010). IBGE. Instituto Brasileiro de Geografia e Estatística. Censo demográfico, 2010. Recuperado em 02 dezembro, 2015, de: http://www.ibge.gov.br/home/.

Camarano, A. A., Kanso, S., Mello, J. L., \& Pasinato, M . T. (2004). Famílias: espaço de compartilhamento de recursos e vulnerabilidades. In: Camarano, A. A. Os Novos Idosos Brasileiros: Muito Além dos 60?, 137-165. Rio de Janeiro, RJ: IPEA. Recuperado em 02 dezembro, 2015, de: http://www.mestradoprofissional.gov.br/portal/index.php?option=com _content\&view $=$ article\&id $=5476$.

Camargos, M. C. S., Rodrigues, R. N., \& Machado, C. J. (2009). Expectativa de vida saudável para idosos brasileiros, 2003. Ciênc. Saúde Coletiva, 14(5), 1903-1909. Recuperado em 02 novembro, 2015, de: http://www.scielo.br/pdf/csc/v14n5/32.pdf.

Costa, F. F. da Silva, \& Lodovici, F. M. M. (2016). O cuidador familiar de idosos em cuidados paliativos: limites e possibilidades. $O$ Envelhecimento Ativo e seus fundamentos, 33-65. São Paulo, SP: Portal Edições/PUC-SP.

Edmonds, E., Mammen, K., \& Miller, D. L. (2005). Rearranging the family? Income support and elderly living arrangements in a low-income country. Journal of Human Resources, 40, 186-207. Recuperado em 02 novembro, 2015, de: http://www.nber.org/papers/w10306.

Freitas, R. de, Santos, S. S. C., Hammerschmidt, K. S. de A., Silva, M. E. da, \& Pelze, M. T. (2011). Cuidado de enfermagem para prevenção de quedas em idosos: proposta para ação. Rev. Bras. Enferm, 64(3), 478-485. Recuperado em 02 novembro, 2015, de: http://www.scielo.br/pdf/reben/v64n3/v64n3a11.pdf. 
Giacomin, K. C., Uchoa, E., \& Lima-Costa, M. F. F. (2005). Projeto Bambuí: a experiência do cuidado domiciliário por esposas de idosos dependentes. Cad. Saúde Pública, 21(5), 1509-1518. Recuperado em 02 novembro, 2015, de: http://www.scielo.br/pdf/\%0D/csp/v21n5/24.pdf.

Ghosh, S. (2007). Living arrangements among elderly women in India. In: Seminar on ageing in developing countries: building bridges for integrated research agendas. Anais... Santiago, Chile. Recuperado em 01 junho, 2015, de: http://www.iussp.org/members/restricted/publications/Santiago07/programme07.php.

Gratão, A. C. M., Talmelli, L. F. da S., Figueiredo, L. C., Rosset, I., Freitas, C. P., \& Rodrigues, R. A. P. (2013). Dependência funcional de idosos e a sobrecarga do cuidador. Revista da Escola de Enfermagem da USP, 47(1), 137-144. Recuperado em 02 novembro, 2015, de: http://www.scielo.br/pdf/reeusp/v47n1/a17v47n1.pdf.

Madeira, M. C., Siqueira, F. C. V., Facchini, L. A., Silveira, D. S. da, Tomasi, E., Thumé, E., Silva, S. M., Dilélio, A., \& Piccini, R. X. (2013). Atividade física no deslocamento em adultos e idosos do Brasil: prevalências e fatores associados. Cad. Saúde Pública,29(1), 165-174. Recuperado em 02 novembro, 2015, de: http://www.scielo.br/pdf/csp/v29n1/19.pdf.

Marteleto, L. (2007). Demographic change and the living arrangements of the elderly: the case of Brazil. In: Population Association of America (PAA) annual meeting. Proceedings. Nova York.

Monteiro, L. M., \& Sena, T. C. C. B. (2012). Análise socioeconômica dos arranjos familiares em um CRAS no município de Belém, PA: o papel do idoso no contexto familiar. Revista Portal de Divulgação, 27. Recuperado em 02 novembro, 2015, de: file:///C:/Users/Dados/Downloads/303-303-1-PB.pdf.

Mourão, A. R. de C., Novais, F. V., Andreoni, S., \& Ramos, L. R. (2013). Atividade física de idosos relacionada ao transporte e lazer. Rev. Saúde Pública, 47(6), 1112-1122. Recuperado em 02 novembro, 2015, de: http://www.scielo.br/pdf/rsp/v47n6/0034-8910rsp-47-06-01112.pdf.

Oliveira, P. B., \& Tavares, D. M. S. (2014). Condições de saúde de idosos residentes em Instituição de Longa Permanência segundo necessidades humanas básicas. Rev. Bras. Enferm, 67(2), 241-246. Recuperado em 02 novembro, 2015, de: http://www.scielo.br/pdf/reben/v67n2/0034-7167-reben-67-02-0241.pdf.

Oliveira, M. C., Boaretto, M. L., Vieira, L., \& Tavares, K. O. (2014). Percepção do cuidador familiar de idosos dependentes sobre o papel do profissional da saúde em sua atividade. Semina: Ciências Biológicas e da Saúde, 35(2), 81-90. Recuperado em 02 novembro, 2015, de: http://www.uel.br/revistas/uel/index.php/seminabio/article/view/19157.

Santos, A. A., \& Pavarini, S. C. I. (2012). Funcionalidade familiar de idosos com alterações cognitivas: a percepção do cuidador. Rev. Esc. Enferm. USP, 46(5), 1141-1147. Recuperado em 02 novembro, 2015, de: http://www.scielo.br/pdf/reeusp/v46n5/15.pdf.

Santos, V. C. R., Kalsing, A., Ruiz, E. N. F., Roese, A., \& Gerhardt, T. E. (2013). Perfil das internações por doenças crônicas não-transmissíveis sensíveis à atenção primária em idosos na Metade Sul do RS. Rev Gaúcha Enferm, 34(3), 124-131. Recuperado em 02 novembro, 2015, de: http://www.scielo.br/pdf/rgenf/v34n3/a16v34n3.pdf. 
Santos, N. M. F., \& Tavares, D. M. S. (2012). Correlação entre qualidade de vida e morbidade do cuidador de idoso com acidente vascular encefálico. Rev. Esc. Enferm. USP, 46(4), 960-966. Recuperado em 02 novembro, 2015, de: http://www.scielo.br/pdf/reeusp/v46n4/25.pdf.

Smith, A. de A. F., Costa, L. S. da, Oliveira, M. F. de, Silva, A. O., Caliri, M. H. L, \& Silva, L. M. (2011). Teoria das representações sociais no âmbito do envelhecimento: uma revisão integrativa. Rev. Pesq.: Cuidado é fundamental (Número Supl.), 242-250. Recuperado em 02 novembro, 2015, de: file://C:/Users/Dados/Downloads/1990-101931-PB.pdf.

Vieira, J. C., Massoni, A. C. de L. T., Freitas, C. H. S. de M., Marinho, F. B., \& Costa, C. H. M. da. (2011). Um olhar sobre os cuidadores de idosos de instituições geriátricas de João Pessoa, Paraíba: perfil e cuidados com a saúde bucal dos idosos. Rev. Bahiana de Saúde Pública, 35(3), 604-618. Recuperado em 02 novembro, 2015, de: http://inseer.ibict.br/rbsp/index.php/rbsp/article/view/281.

Recebido em 21/07/2016

Aceito em 30/12/2016

Luisa Kecyane Batista Cardoso - Discente do Programa de Pós-Graduação em Enfermagem e Saúde, nível Mestrado, Universidade Estadual do Sudoeste da Bahia, UESB, Jequié, BA, Brasil.

E-mail: keicyluisa@hotmail.com

Talita Santos Oliveira Sampaio - Fisioterapeuta. MS. em Ciências da Saúde, Programa de Pós-Graduação em Enfermagem, Universidade Estadual do Sudoeste da Bahia, UESB. Professora Titular da Faculdade Independente do Nordeste, FAINOR.

E-mail: talitafisio@gmail.com

Alba Benemérita Alves Vilela - Enfermeira. Ph.D. em Enfermagem. Professora plena do Departamento de Saúde (DS) e do Programa de Pós-Graduação em Enfermagem e Saúde, Universidade Estadual do Sudoeste da Bahia, UESB. Jequié, Bahia, Brasil. E-mail: albavilela@gmail.com 